\title{
PANORAMA E IMPORTÂNCIA ECONÔMICA DO CAFÉ NO MERCADO INTERNACIONAL DE COMMODITIES AGRÍCOLAS: UMA ANÁLISE ESPECTRAL
}

\author{
André Cutrim Carvalho'; David Ferreira Carvalho²; Gisalda Carvalho Filgueiras³; Ana Cláudia de Sousa Araújo4; \\ Abner Vilhena de Carvalho ${ }^{5}$ \\ ${ }^{1}$ Professor (Pós-)Doutor da FACECON/ICSA/UFPA e do PPGEDAM/NUMA/UFPA, Belém, Pará, Brasil, \\ andrecc83@gmail.com \\ 2 Professor (Pós-)Doutor da FACECON/ICSA/UFPA, Belém, Pará, Brasil, david.fcarvalho@yahoo.com.br \\ 3 Professora (Pós-)Doutora da FACECON/ICSA/UFPA, Belém, Pará, Brasil, gisalda.filgueiras@gmail.com \\ ${ }^{4}$ Mestranda pelo Programa de Química Medicinal e Modelagem Molecular da UFPA, Belém, Pará, Brasil, \\ nana22araujo@gmail.com \\ ${ }^{5}$ Doutorando pelo Programa Sociedade, Natureza e Desenvolvimento da UFOPA, Santarém, Pará, Brasil, \\ abnervilhena@hotmail.com
}

RESUMO: Este artigo procura discutir o panorama e a importância econômica do café para o Brasil ao fazer uso do instrumental empírico e analítico da análise espectral, tendo como base a série temporal dos preços do café no mercado internacional de commodities agrícolas de Nova lorque (EUA). De fato, o café é um importante componente das exportações totais de vários países, inclusive do Brasil. Com o passar do tempo, o café acabou se tornando um produto, economicamente, importante de muitas famílias pelo mundo. Do ponto de vista metodológico, a pesquisa utilizou o arcabouço estatístico-econométrico oriundo da análise espectral de séries temporais. Os resultados obtidos dessa análise evidenciam que os choques climáticos sobre os preços do café poderão ter efeitos mais longos e que políticas agrícolas, que afetem diretamente a cadeia produtiva do café, poderão ter efeitos mais duradouros. Além disso, os resultados indicam que a tendência dos preços do café se configura numa combinação de tendências dos tipos determinística e estocástica.

PALAVRAS-CHAVE: Análise Espectral, Brasil, Café, Commodities Agrícolas.

\section{PANORAMA AND ECONOMIC IMPORTANCE OF COFFEE IN THE INTERNATIONAL MARKET FOR AGRICULTURAL COMMODITIES: A SPECTRAL ANALYSIS}

\begin{abstract}
This paper aims to discuss the panorama and importance of coffee for Brazil, especially in economic terms, by making use of the empirical and analytical instruments of the spectral analysis, based on the time series of coffee prices in the international agricultural commodities market. New York (USA). In fact, coffee is an important component of total exports from several countries, including Brazil. Over time, coffee has become an economically important product of many families around the world. From the methodological point of view,
\end{abstract}

Agroecossistemas, v. 9, n. 2, p. 223 - 222, 2017, ISSN online 2318-0188 
the research used the statistical-econometric framework derived from the spectral analysis of time series. The results of this analysis show that climate shocks on coffee prices may have longer effects and those agricultural policies that directly affect the coffee production chain may have longer lasting effects. Moreover, the results indicate that the coffee price trend is in a combination of deterministic and stochastic trends.

KEYWORDS: Agricultural Commodities, Brazil, Coffee, Spectral Analysis.

\section{PANORAMA E IMPORTANCIA ECONOMICA DEL CAFÉ EN EL MERCADO INTERNACIONAL DE COMMODIDADES AGRÍCOLAS: UN ANÁLISIS ESPECTRAL}

RESUMEN: Este artículo busca discutir el panorama y la importancia económica del café para Brasil al hacer uso del instrumental empírico y analítico del análisis espectral, teniendo como base la serie temporal de los precios del café en el mercado internacional de commodities agrícolas de Nueva York ). De hecho, el café es un importante componente de las exportaciones totales de varios países, incluso de Brasil. Con el paso del tiempo, el café acabó convirtiéndose en un producto, económicamente, importante de muchas familias por el mundo. Desde el punto de vista metodológico, la investigación utilizó el marco estadístico-econométrico oriundo del análisis espectral de series temporales. Los resultados obtenidos de este análisis ponen de manifiesto que los choques climáticos sobre los precios del café pueden tener efectos más largos y que las políticas agrícolas que afecten directamente a la cadena productiva del café pueden tener efectos más duraderos. Además, los resultados indican que la tendencia de los precios del café se configura en una combinación de tendencias de los tipos determinista y estocástico.

PALABRAS CLAVE: Análisis Espectral, Brasil, Café, Commodities Agrícolas.

\section{INTRODUÇÃO}

Desde os primórdios das transações mercantis simples, o café tem sido um produto de extrema relevância nas economias mundiais. Como recordam Dias e Silva (2015, p. 87), "o café chegou ao Brasil em 1727, na cidade de Belém, então província do Grão-Pará, trazido da Guiana Francesa por Francisco de Melo Palheta. Seu plantio se desenvolveu no interior de São Paulo e Rio de Janeiro, onde prosperou no vale do Paraíba". Sobre isso, Cunha et al. (2010, p. 516) destacam a relevância do café nos seguintes termos: 
O café é um dos produtos agrícolas de maior valor e dinamismo comercial. Seu mercado foi caracterizado, ao longo do século XX, por uma série de intervenções. Inicialmente, as ações eram conduzidas pelos países produtores, já que obtinham grande parte de sua renda da economia cafeeira. O Brasil tinha papel de destaque nesse processo e, até meados do século, atuou diretamente no processo de formação dos preços externos (em razão de sua elevada parcela de mercado, que, nas primeiras décadas de 1900, chegou a 75\% da produção mundial).

De acordo com a base de dados da Associação Brasileira da Indústria de Café $(A B I C)$, parceria do Consórcio Pesquisa Café, coordenado pela Embrapa Café, ano 2015, o consumo interno de café beneficiado no Brasil passou de 20,085 milhões de sacas de $60 \mathrm{~kg}$ para 20,333 milhões de sacas. Assim, o consumo per capita também aumentou ligeiramente no período, subindo de 4,87 kg/habitante/ano para 4,89 kg/habitante/ano de café torrado e moído e de 6,09 kg de café verde em grão para $6,12 \mathrm{~kg}$, o que equivale a aproximadamente 81 litros/habitante/ano.

Estima-se que entre 20 a 25 milhões de famílias, em mais de 50 nações em desenvolvimento, e em mais de 5 milhões de fazendas, produzem e comercializam o café. O café é uma planta na forma de um arbusto lenhoso, perene, pertencente à família da Rubiaceae que possui próximo de 500 gêneros e mais de 6000 espécies, ou seja, um importante produto - do ponto de vista econômico - de várias famílias pelo mundo.

Destas várias, duas espécies são amplamente comercializadas: o café arábica, e o café robusta. O café arábica chamado simplesmente de arábica, que responde por cerca de $60 \%$ a $70 \%$ de produção mundial. O café arábica produz boas bebidas de sabor muito apreciado e ótimo aroma, porém é mais suscetível aos ataques de pragas e doenças, sendo que a expansão geográfica do café arábica está concentrada na América Latina, na América Central, na África Oriental, na Índia e, até certo ponto, na Indonésia.

Em contrapartida, o café robusta responde por 30\% a 40\% de produção mundial. Robusta é o nome da variedade do Coffea canéfora, sendo oriundo de uma árvore pequena - até 10 metros em altura que pode crescer em altitudes mais baixas que arábica, com rendimentos mais altos e com certa resistência para doença. Os 
frutos, porém, são de gostos inferiores ao do café arábica, e tem duas vezes mais o conteúdo de cafeína.

A cultivar robusta, geralmente, tem preço mais baixo no mercado, e é frequentemente usada para café solúvel, ou para reforçar a cafeína dos "cafés expressos". O cultivar robusta vem se expandido na África Central, na Ásia e na América Latina, sobretudo no Brasil, onde é conhecida como "Conillon" e é muito importante para a economia local.

Dito isso, o presente artigo pretende utilizar o instrumental empírico-analítico da análise espectral. Wolff et al. (2011, p. 03) define série temporal como sendo "uma coleção de observações feitas sequencialmente ao longo do tempo". E prossegue "a análise espectral de séries temporais estacionárias decompõe os dados da série temporal em componentes senoidais com coeficientes aleatórios nãocorrelacionados".

Além dessa decomposição, há uma correspondente decomposição da função de auto covariância, a qual segundo Mattos e Ferreira (2016, p. 01):
Uma série temporal, segundo a decomposição clássica, pode ser decomposta em quatro componentes não observáveis: tendência, sazonalidade, ciclo e erro. A sazonalidade, objeto de estudo deste artigo, é causada por movimentos oscilatórios de mesma periodicidade que ocorrem em período intra-anual, como variações climáticas, férias, feriados, entre outros.

Assim, o principal objetivo do presente trabalho é discorrer sobre o panorama e a importância econômica do café para o Brasil ao fazer uso do instrumental da análise espectral, tendo como base para isso a série temporal dos preços do café no mercado internacional de commodities agrícolas de Nova lorque nos EUA.

\section{MATERIAL E MÉTODOS}

Conforme Esquivel et al. (2012, p. 88), "o desenvolvimento de métodos estatísticos para análise de dados obtidos em situações em que as observações são dependentes tem apresentado crescimento vertiginoso nas últimas décadas". A técnica que será utilizado envolve a análise estrutural de séries temporais, que consiste na decomposição da série em termos dos seus componentes não-observáveis (estruturais) 
de tendência, sazonalidade, cíclica e volatilidade (GUJARATl; PORTER, 2011; SARTORIS, 2013).

Outro método importante é o da análise espectral. A análise espectral de séries é, de certo modo, uma variante estilizada da análise de séries temporais que fornece informações complementares àquelas apropriadas às análises clássicas de análise temporal, sobretudo da ocorrência do comportamento cíclico nas séries. Para Esquivel et al. (2012, p. 89):

Os métodos estatísticos clássicos para análise de séries temporais encontram-se bem documentados na literatura pertinente. Contudo, boa parte desses métodos requer conhecimento especializado para sua correta aplicação. Sendo assim, o uso adequado dos modelos clássicos exigirá verificações das suas suposições, o que demanda esforços e experiência, na análise exploratória dos dados.

O fundamento básico da análise espectral de uma série consiste na representação de uma série temporal $\left\{\mathrm{Z}_{\mathrm{t}}\right\}_{\mathrm{t}=-\infty}^{\infty}$ como uma média ponderada de funções periódicas do tipo seno (w) e cosseno (w), em que w representa uma frequência particular; e também na determinação da importância dos ciclos de distintas frequências, caso eles existam, para explicar a variância de $\left\{\mathrm{Z}_{\mathrm{t}}\right\}_{\mathrm{t}=-\infty}^{\infty} \mathrm{e}$, por conseguinte, o comportamento da série temporal.

Quando se considera uma dada variável econômica, com distribuição aleatória ao longo do tempo, tem-se uma série temporal, $\left\{Z_{t}\right\}_{t=1}^{T}$, em que T é o número de observações (realizações) da série, que é uma amostra de um processo estocástico infinito do tipo: $\left\{Z_{t}\right\}_{t=-\infty}^{\infty}$

Chiesa e Miguel (2017, p. 32) afirmam que "no desenvolvimento probabilístico para vibração, tanto a excitação como a resposta estrutural são modeladas como processos estocásticos". E continua afirmando que "um processo estocástico pode ser especificado em termos de um número relativamente pequeno de parâmetros estatísticos e funções, como a função densidade espectral de potência, também denominada espectro de potência".

Desta forma, suponhamos a expressão dada por $\left\{\mathrm{Z}_{\mathrm{t}}, \mathrm{t} \in \mathrm{Z}\right\}$, como representando 
um processo estacionário com média zero e função de auto covariância satisfazendo uma condição de independência assintótica - no sentido de que os valores do processo bastante separados no tempo sejam pouco dependentes - que pode ser expresso do seguinte modo:

$$
\sum_{\tau=-\infty}^{\infty}|\gamma(\tau)|<\infty
$$

Neste caso, Morettin e Toloi (2004) e, também, Chiesa e Miguel (2017) observam que nessa condição a função densidade espectral, ou espectro de $\mathbf{Z}_{\mathbf{t}}$, é definida como uma transformada de Fourier de:

$$
f(\lambda)=\frac{1}{2 \pi} \sum_{\tau=-\infty}^{\infty} \gamma(\tau) e^{i \lambda \tau}, \quad-\infty \leq \lambda \leq \infty
$$

Em que: $e^{i \lambda}=\cos \lambda+i \operatorname{sen} \lambda$ e $i=\sqrt{-1}$. A função densidade, $\mathrm{f}(\lambda)$, é limitada, nãonegativa e uniformemente contínua, além de $f(\lambda)$ ser par e periódica do período $2 \pi$. Este teorema pode ser assim demonstrado:

$\left.1^{\circ}\right) \bigcirc$ fato da função densidade $f(\lambda)$ ser limitada segue da expressão (1) e também da expressão $|f(\lambda)| \leq \frac{1}{2 \pi} \sum_{\tau=-\infty}^{\infty} \mid \lambda(\tau)$;
20) Da diferença: $\Delta \mathrm{f}(\lambda)=$ $|f(\lambda+w)-f(\lambda)| \leq \frac{1}{2 \pi} \sum_{\tau=-\infty}^{\infty}|\gamma(\tau)|\left|e^{-i w \tau}-1\right|$, tem-se que está última tende para zero quando $w \rightarrow 0$, independente de $\lambda$; o que prova que a função densidade da série é uniformemente contínua;

30) Como a função de auto covariância, facv $\lambda(\tau)$, é par, segue-se que o espectro também é par e, portanto, real;

$\left.4^{\circ}\right)$ Como a função densidade, $\mathrm{f}(\lambda)$, é periódica de período $2 \pi$, basta considerarmos o intervalo $|-\pi, \pi|$; e

$\left.5^{\circ}\right)$ Como a função densidade, $f(\lambda)$, é par, basta representá-la no intervalo $[0, \pi]$.

A partir da expressão (2) segue, em termos de variáveis contínuas, uma outra para a função densidade, $\lambda(\tau)$, representada por:

$$
\lambda(\tau)=\int_{-\pi}^{\pi} f(\lambda) d \lambda \quad \tau \in Z,
$$

Esta função densidade contínua, $\lambda(\tau)$, pode ser, por assim dizer, "recuperada" da função densidade discreta $\mathrm{f}(\lambda)$. Assim, sob a ótica da quantidade de informação probabilística que fornecem, o espectro e a 
função de auto covariância são ferramentas equivalentes.

Ademais, a equação (3) expressa a facv $\lambda(\tau)$ de uma série estacionária satisfazendo (1), igualmente como os coeficientes da análise de Fourier da função densidade $\mathrm{f}(\lambda)$, conforme Morettin e Toloi (2004). A função de auto covariância em geral é de valor imprescindível para descrever a estrutura do processo estocástico.

Na análise do domínio de frequência existe, além da Função de Densidade Populacional, a Função de Distribuiç̧ão Espectral que é a representação espectral da função de auto covariância. Assim, o Espectro Populacional de um processo estocástico do tipo: $\left\{\mathrm{y}_{\mathrm{t}}\right\}_{\mathrm{t}=-\infty}^{\infty}$, pode ser representado como:

$$
f_{y}(\omega)=\frac{1}{2 \pi}\left[y_{0}+2 \sum_{j=1}^{\infty} y_{j} \cos (\omega j)\right]
$$

Ressalta-se que, como o espectro populacional representa uma função contínua de $\omega$, definida no conjunto dos números reais, e $\mathrm{y}_{\mathrm{j}}$ representa as auto covariâncias de um processo estacionário, então a equação (4) terá um valor positivo para qualquer $\omega$ (GUJARATI; PORTER, 2011).

Tal como acontece com a análise do correlograma, na análise espectral, Lamounier e Leite (2004, p. 624) afirmam que "é o gráfico do espectro que fornece as informações necessárias sobre a variância de $y_{t}$ e quais frequências afetam $o$ comportamento cíclico da série temporal considerada". No entanto, se o gráfico da função $\mathrm{f}_{\mathrm{y}}(\omega)$ não apresentar 'picos', ou seja, isso quer dizer se o gráfico dessa função for plano, implica que a série temporal considerada não possui variações cíclicas, logo pode ser caracterizada como um ruído branco (LAMOUNIER; LEITE, 2004). Sobre isso, Brunato et al. (2016, p. 08) observam:

Primeiramente, os resíduos do modelo não devem ser correlacionados. Essa condição pode ser analisada por meio dos correlogramas, onde os resíduos devem ter as características de um ruído branco, ou seja, os valores plotados não ultrapassam os limites de controle do correlograma.

Para verificar se um espectro de uma dada série tem 'picos' estatisticamente 
significativos, pode-se recorrer a dois métodos, a saber: i) o primeiro refere-se à análise visual a partir da função densidade espectral estimada através da identificação dos picos que mais se destacam e; ii) o segundo diz respeito ao emprego de um teste de hipótese (nula) de que o espectro estimado não é estatisticamente diferente do espectro ruído branco, o que pode ser feito comparando os valores estimados obtidos do periodograma por intermédio de uma distribuição exponencial (LAMOUNIER; LEITE, 2004).

Como nas análises empíricas de séries temporais, o pesquisador dispõe apenas das amostras finitas da população, não se pode estimar o número infinito de parâmetros da população expressa por: $\left\{\mathrm{y}_{\mathrm{t}}\right\}_{-\infty}^{\infty}$. Neste caso, deve-se operacionalizar a análise espectral com um conceito análogo ao do espectro populacional, para a análise espectral dos dados amostrados, que é o de periodograma amostral.
A fórmula geral dos estimadores consistentes do espectro $\mathrm{f}_{\mathrm{y}}(\omega)$ é dada pela expressão:

$$
f_{y}(\omega)=\frac{1}{2 \pi}\left(\lambda_{0} c_{0}+2 \sum_{j=1}^{M_{T}} \lambda_{j} c_{j} \cos \omega_{j}\right)
$$

Lamounier e Leite (2004) observam que essa sequência dos pesos $\lambda_{\mathrm{j}}=\lambda_{0,} \lambda_{1}, \lambda_{2}, \ldots \ldots, \lambda_{\mathrm{M}_{\mathrm{T}}}$ é chamada de janela de defasagem, e o termo $M_{T}(<N)$ de ponto de truncamento. A escolha do valor do ponto de truncamento, $\boldsymbol{M}_{T}$, é dada por $M_{T}=2 \sqrt{N}$, sendo $\mathrm{N}$ o número de valores da série temporal amostrada.

Há, ainda, várias formas de representações espectrais de um processo estacionário $\left\{Z_{t}, t \in R\right\}$, real, de média zero e facv $\lambda(\tau)$, suposta contínua para todo $\tau$. Para fins deste trabalho, entretanto, a análise espectral para verificação de ciclos numa série temporal $\mathbf{Z}_{\mathrm{t}}$ tem como ponto de partida o Teorema da Representação Espectral de Crámer e Leadbetter (1967)

\footnotetext{
${ }^{1}$ O teorema em questão não será demonstrado, pois foge do escopo do artigo.
} 
Assim, se há um processo estocástico estacionário que satisfaça as condições do tipo: $\{U(\lambda), \lambda \in R\}$, então ele é processo estocástico de incrementos ortogonais:

$$
Z_{t}=\int_{-\infty} e^{i t \lambda} d U(\lambda), \quad \mathrm{t} \in \mathrm{R}
$$

Em que $\mathrm{R}$ representa o conjunto dos números reais. Este processo estocástico do tipo: $U(\lambda)$, chamado de processo espectral associado a $\mathrm{Z}(\mathrm{t})$, tem as seguintes propriedades:

a) $E[d U(\lambda)]=0$, para todo $\lambda_{i}$ b) $E[d U(\lambda)]^{2}$, para todo $\lambda_{i} \quad$ e $\quad$ c) $\operatorname{Cov}\left[d U(\lambda), d U\left(\lambda^{\prime}\right)\right]=E\left[d U(\lambda) d U\left(\lambda^{\prime}\right)\right]=0$, $\lambda \neq \lambda^{\prime}$.

Assim, se tivermos um processo estacionário discreto, $\left\{Z_{t}, t \in Z\right\}$, então, tem-se:

$$
Z_{t}=\int_{-\pi}^{\pi} e^{i t \lambda} d U(\lambda), \mathrm{t} \in \mathrm{Z} .
$$

Sendo $U(\lambda)$ um processo estocástico estacionário discreto com as mesmas propriedade acima, somente que $\lambda \in[-\pi, \pi] . \quad$ Mas, um processo estacionário discreto, $\mathrm{y}_{\mathrm{t}}$, com frequências pertencentes ao intervalo $[0, \pi]$, pode ser representado assim:

$$
y_{t}=\mu+\int_{0}^{\pi}[\alpha(\omega) \cdot \cos (\omega t)] d \omega+\int_{0}^{\pi}[\beta(\omega) \cdot \operatorname{sen}(\omega t)] d \omega
$$

$$
\text { A equação (7) seria a forma mais }
$$
frequentemente utilizada para expressar a representação Espectral do Processo Estocástico Estacionário $y_{\mathrm{t}}$. É da equação (5) que surge o conceito de análise espectral que descreve o valor de uma variável $\mathrm{y}_{\mathrm{t}}$ como sendo uma somatória de funções periódicas da forma $\cos (\omega t)$ e $\operatorname{sen}(\omega t)$, em que $\omega$ representa uma frequência particular no intervalo $[0, \pi]$.

Além disso, a frequência angular $\omega=\pi$, medida em termos de radianos por unidade de tempo, é conhecida como frequência de Nyquist e indica o valor mais elevado com que se pode obter informações sobre ciclos periódicos de séries temporais. Neste contexto, a equação (5) denota que cada frequência no intervalo $[0, \pi]$ pode auxiliar na variação dos valores de uma série temporal. 


\section{RESULTADOS E DISCUSSÃO}

Até o início da crise internacional do café dos anos 90, ele representava a segunda mercadoria mais negociada na economia mundial. Na verdade, Rego e De Paula (2012, p. 02) observam que "a crise de café começou com o preço internacional despencando em 1988 para um nível abaixo do custo de produção. A persistência dos preços baixos do café resultou em queda dos investimentos e redução da renda dos produtores rurais".

Porém, a partir de 2000, o valor negociado do café no mercado internacional alcançou a cifra de US\$5,6 bilhões em detrimento de US\$ 12 bilhões atingidos anos atrás. De fato, o valor de mercado de varejo do café é de longe mais alto que o valor do mercado atacadista de café verde, com estimativas anuais recentes de US\$ 55 bilhões, valor este que os países exportadores se apropriam de apenas 15\%, como demonstram os dados da International Coffee Organization (ICO) de 2010.

O consumo mundial de café é, em média, cerca de mais de 6 milhões de toneladas por ano. A Europa é ainda o maior mercado, seguido pelo EUA e o Japão, segundo a International Coffee Organization (ICO).
Estima-se que sejam consumidos no mundo cerca de 28 milhões de sacos de café de $60 \mathrm{~kg}$ por ano, mas como toda commodity agrícola, o café apresenta flutuações nos seus preços internacionais por diversas razões, sobretudo climáticas (secas e geadas) e da política de comércio exterior.

Além de flutuações cíclicas causadas por choques climáticos, mais recentemente a economia do café vem passando por profundas mudanças estruturais, com reflexos no mercado internacional, devido os avanços tecnológicos e a emergência de novos produtores no cenário mundial que estão redefinindo uma nova agenda que inclui:

10) Liberalização do mercado em muitos países produtores significou uma evolução dos sistemas de marketing de café;

$2^{\circ}$ ) A natureza de provisão, particularmente no que se relaciona a aumentos da quantidade e da qualidade dos cafés brasileiros e vietnamitas;

30) Adaptação de tecnologia às novas áreas, acrescentando o uso de custo produção e de transação mais baixo para os cafés naturais, arábicas e robustas; e 
$\left.4^{0}\right)$ Flexibilidade pela inserção dos blends (misturas de café) como resposta a oferta de custo mais baixo.

Nishijima et al. (2012, p. 73) adverte que o crescimento da produção de café tem sido impulsionado por vários fatores, com destaque para:

(i) aumento dos preços, decorrente principalmente de problemas climáticos, já que a demanda tende a crescer de forma estável; (ii) políticas agrícolas das nações produtoras e dos países desenvolvidos visando o combate à pobreza em países pobres; (iii) inovação tecnológica, particularmente com técnicas de fertirrigação que têm resultado em melhora da produtividade; e (iv) desvalorização cambial nos países produtores.

Nota-se que a maioria de comércio internacional do café consiste do café verde (maduro) transportado em sacos de $60 \mathrm{~kg}$ ou em recipientes para o transporte em granel. Essencialmente, dois conjuntos de preços estão disponíveis para o café: $1^{\circ}$ ) os preços que servem como indicadores do volume do comércio físico, e onde o contrato se refere à qualidade específica, origem, remessa, moeda corrente e destino do café; e $2^{\circ}$ ) os preços determinados pelos mercados futuros que refletem o consumo da produção com base nos princípios de mercado (e ações subjacentes) e fatores técnicos de comércio.

Além disso, sob condições normais de fornecimento do café, os preços do mercado internacional são mais altos para os cafés milds colombiano, seguido por outros milds, arábicas e robustas brasileiros. Durante um certo tempo, Brasil e Colômbia foram os principais produtores mundiais de café, mas esta situação mudou nos anos 90 e, principalmente, no ano 2000 com o crescimento de produção de café do Vietnã, que substituiu a Colômbia como o segundo maior produtor de café do mundo em 1999/2000.

Há, ainda, uma discussão sobre o papel do café verde no mercado. Em linhas gerais, o café verde está disponível para demandantescompradores diretamente da fonte produtora, ou via os grandes atacadistas reexportados dos EUA e da Europa, e o modelo de comércio dominante para a indústria de café está avaliando o comércio internacional baseado em dois padrões praticados pela Diretoria de Comércio de Nova Iorque e pelo Mercado Internacional Financeiro e de Futuros de Londres. 
Ao mesmo tempo, os mercados futuros do café são um modo de administrar riscos estabelecendo uma base de preço do café para o produtor, exportador, expedidor e consumidor. No início, só existiam nos principais centros comerciais as companhias de café, porém, recentemente, com a presença ativa dos capitais de investimentos de risco, o crescimento dos mercados futuros passou a transacionar risco, o que adiciona volatilidade ao preço internacional do café.

Na atual conjuntura, porém, os países produtores são responsáveis por cerca de 60\% da produção mundial total como pode ser constatado através da Tabela 1.

Tabela 1. Produção dos países exportadores de café: 2000-2006/1000 sacos.

\begin{tabular}{l|c|c|c|c|c|c}
\hline Anos & 2000 & 2001 & 2002 & 2003 & 2004 & 2006 \\
\hline Produção mundial & 116.619 & 108.188 & 123.430 & 105.857 & 115.444 & 108.222 \\
\hline Grandes Produtores* & 106.765 & 100.496 & 114.817 & 97.485 & 107.326 & 99.498 \\
\hline Brasil & 34.100 & 30.837 & 48.617 & 28.787 & 39.273 & 32.944 \\
\hline Camarões & 1.113 & 686 & 801 & 900 & 727 & 1.000 \\
\hline Colômbia & 10.532 & 11.999 & 11.889 & 11.197 & 12.042 & 11.000 \\
\hline Costa Rica & 2.293 & 2.127 & 1.893 & 1.783 & 1.887 & 1.778 \\
\hline Costa do Marfim & 4.846 & 3.595 & 3.145 & 2.689 & 2.328 & 2.171 \\
\hline Equador & 872 & 893 & 732 & 766 & 938 & 1.125 \\
\hline El Salvador & 1.752 & 1.686 & 1.438 & 1.477 & 1.438 & 1.372 \\
\hline Etiópia & 2.768 & 3.756 & 3.693 & 3.874 & 5.000 & 4.500 \\
\hline Guatemala & 4.940 & 3.669 & 4.070 & 3.610 & 3.703 & 3.675 \\
\hline Honduras & 2.667 & 3.036 & 2.497 & 2.968 & 2.575 & 2.990 \\
\hline Índia & 4.516 & 4.970 & 4.683 & 4.495 & 3.844 & 4.630 \\
\hline Indonésia & 6.978 & 6.833 & 6.785 & 6.571 & 7.536 & 8.340 \\
\hline Quênia & 1.001 & 991 & 945 & 673 & 709 & 1.002 \\
\hline México & 4.815 & 4.200 & 4.000 & 4.550 & 3.407 & 4.200 \\
\hline Nicarágua & 1.595 & 1.116 & 1.199 & 1.546 & 1.130 & 1.718 \\
\hline Papua-Nova Guiné & 1.041 & 1.062 & 1.085 & 1.155 & 997 & 1.267 \\
\hline Peru & 2.596 & 2.749 & 2.900 & 2.616 & 3.355 & 2.420 \\
\hline Uganda & 3.401 & 3.158 & 2.890 & 2.598 & 2.593 & 2.366 \\
\hline Vietnã & 14.939 & 13.133 & 11.555 & 15.230 & 13.844 & 11.000 \\
\hline Pequenos Produtores** & 9.854 & 7.692 & 8.613 & 8.372 & 8.118 & 8.724 \\
\hline Fonte: Intrin
\end{tabular}

Fonte: International Coffee Organization (2010). * Grandes produtores: produzem um milhão ou mais sacos de $60 \mathrm{~kg}$ de café. ${ }^{* *}$ Pequenos produtores: produzem menos de um milhão de sacos de $60 \mathrm{~kg}$ de café. 
Isto acontece pelo fato de que o volume do café negociado nos mercados futuros excede a produção global do café por um fator de dez, onde o desafio imediato para a indústria de café é como sustentar as melhores condições de mercado para evitar a volta dos ciclos violentos prejudiciais a economia do café. Entretanto, parece ser pouco evidente no cenário atual uma recuperação do preço internacional do café no nível dos anos anteriores à crise, devido o problema do princípio da provisão permanente.

Os preços dos cafés mild arábica colombiano, dos milds arábicas, do arábica natural brasileiro e o robusta brasileiro são negociados no mercado internacional. $\mathrm{Na}$ Tabela 2, no ano de 2004, os preços dos cafés arábica e robusta oscilaram entre os meses de janeiro a dezembro. Já o preço composto ponderado do café verde, que é uma média ponderada dos quatro tipos acima, flutuou entre 58,69 cents/lb (em janeiro) a 77,72 cents/lb (em dezembro).

Em 2005, o preço composto ponderado voltou a subir de 79,35 cents/lb (em janeiro) para 101,44 cents/lb (em março), porém logo em seguida volta a declinar até atingir 86,65 cents/lb em dezembro. Em 2006, o preço composto ponderado começa em alta em janeiro (101,20 cents/lb) e depois declina até chegar no mês outubro em baixa (95,53cents/b).

Neste mesmo período, entretanto, os preços tanto do café arábica quanto do café robusta oscilaram durante $\mathrm{O}$ ano todo, inicialmente como um movimento cíclico ascendente dos preços, entre os meses de fevereiro a junho, e depois com uma dinâmica cíclica descendente de julho a dezembro. No ano de 2006, depois de aumento do preço do café arábica, em janeiro, nota-se uma tendência de queda do preço, nos meses seguintes, sobretudo nos mercados de Nova lorque e da Alemanha.

O café natural arábica brasileiro apresentou uma significativa demanda no mercado mundial que se manifestou pela elevação dos seus preços em 2004, apesar das flutuações de curtíssimo prazo durante alguns meses do ano. Já o café robusta apresentou uma demanda restrita no mercado internacional com a manifestação de uma tendência descendente dos preços e algumas oscilações de subida, sobretudo no mês de junho, mas que não se confirmaram. 
Tabela 2. Cotações dos preços do café arábica e robusta do Brasil no mercado internacional em Nova lorque e na Alemanha: 2004 a 2006.

\begin{tabular}{|c|c|c|c|c|c|c|c|}
\hline \multirow{2}{*}{$\begin{array}{l}\text { Anos/ } \\
\text { Meses }\end{array}$} & \multirow[b]{2}{*}{$\begin{array}{c}\text { Preço } \\
\text { Composto }\end{array}$} & \multicolumn{3}{|c|}{ Arábicas Naturais Brasileiras } & \multicolumn{3}{|c|}{ Robustas } \\
\hline & & $\begin{array}{l}\text { Nova } \\
\text { lorque }\end{array}$ & Alemanha & $\begin{array}{c}\text { Média } \\
\text { Ponderada }\end{array}$ & $\begin{array}{l}\text { Nova } \\
\text { lorque }\end{array}$ & Alemanha & $\begin{array}{c}\text { Média } \\
\text { Ponderada }\end{array}$ \\
\hline 2004 & 62,15 & 68,18 & 69,11 & 68,97 & 37,28 & 35,65 & 35,99 \\
\hline Janeiro & 58,69 & 64,32 & 61,51 & 62,06 & 41,32 & 39,27 & 39,84 \\
\hline Fevereiro & 59,87 & 66,08 & 65,37 & 65,52 & 39,10 & 36,54 & 37,05 \\
\hline Março & 60,80 & 65,79 & 67,26 & 66,97 & 38,61 & 36,22 & 36,70 \\
\hline Abril & 58,80 & 62,89 & 63,89 & 63,70 & 38,02 & 35,96 & 36,37 \\
\hline Maio & 59,91 & 64,31 & 65,52 & 65,16 & 38,04 & 36,19 & 36,56 \\
\hline Junho & 64,28 & 67,62 & 70,06 & 69,61 & 41,09 & 39,54 & 39,87 \\
\hline Julho & 58,46 & 59,39 & 63,77 & 62,89 & 36,44 & 35,90 & 36,02 \\
\hline Agosto & 56,98 & 60,25 & 62,13 & 61,75 & 34,81 & 33,68 & 33,91 \\
\hline Setembro & 61,47 & 69,46 & 68,84 & 68,90 & 35,10 & 34,04 & 34,24 \\
\hline Outubro & 61,10 & 68,63 & 70,23 & 69,91 & 31,77 & 31,65 & 31,67 \\
\hline Novembro & 67,74 & 80,20 & 78,66 & 79,39 & 34,07 & 32,32 & 32,71 \\
\hline Dezembro & 77,72 & 89,17 & 92,08 & 91,76 & 38,98 & 36,49 & 36,92 \\
\hline 2005 & 89,36 & 101,36 & 102,49 & 102,29 & 53,37 & 49,87 & 50,55 \\
\hline Janeiro & 79,35 & 94,00 & 93,56 & 93,63 & 39,63 & 36,30 & 36,96 \\
\hline Fevereiro & 89,40 & 108,05 & 105,58 & 106,11 & 44,61 & 40,40 & 41,24 \\
\hline Março & 101,44 & 117,03 & 120,89 & 120,12 & 50,70 & 49,22 & 49,51 \\
\hline Abril & 98,20 & 112,82 & 114,90 & 114,48 & 53,32 & 50,11 & 50,75 \\
\hline Maio & 99,78 & 111,89 & 115,72 & 114,96 & 58,66 & 55,55 & 56,07 \\
\hline Junho & 96,29 & 105,08 & 107,76 & 107,23 & 62,96 & 59,29 & 60,02 \\
\hline Julho & 88,48 & 94,66 & 97,04 & 96,56 & 60,57 & 57,18 & 57,88 \\
\hline Agosto & 85,31 & 95,66 & 94,81 & 94,98 & 55,60 & 51,07 & 51,97 \\
\hline Setembro & 78,79 & 87,02 & 89,99 & 89,48 & 50,07 & 46,03 & 46,87 \\
\hline Outubro & 82,55 & 94,54 & 94,69 & 94,40 & 50,84 & 46,85 & 47,53 \\
\hline Novembro & 85,93 & 99,35 & 97,63 & 97,96 & 54,72 & 50,78 & 51,45 \\
\hline Dezembro & 86,85 & 96,23 & 97,25 & 97,57 & 58,79 & 55,67 & 56,39 \\
\hline 2006 & 93,75 & 100,97 & 102,22 & 101,97 & 68,60 & 65,13 & 65,72 \\
\hline Janeiro & 101,20 & 115,89 & 114,84 & 114,98 & 66,46 & 62,77 & 63,39 \\
\hline Fevereiro & 97,39 & 109,51 & 108,91 & 109,01 & 65,50 & 62,45 & 62,98 \\
\hline Março & 92,76 & 103,52 & 104,02 & 103,92 & 62,92 & 58,92 & 59,60 \\
\hline Abril & 94,20 & 105,89 & 105,39 & 105,49 & 64,45 & 59,75 & 60,55 \\
\hline Maio & 90,00 & 99,00 & 99,63 & 99,29 & 63,97 & 59,32 & 60,08 \\
\hline Junho & 86,04 & 91,26 & 93,68 & 93,27 & 64,14 & 59,43 & 60,23 \\
\hline Julho & 88,57 & 91,01 & 95,39 & 94,56 & 68,66 & 63,62 & 64,49 \\
\hline Agosto & 95,78 & 98,90 & 100,74 & 100,37 & 75,73 & 73,15 & 73,59 \\
\hline Setembro & 95,98 & 97,36 & 100,06 & 99,53 & 77,88 & 76,95 & 77,11 \\
\hline Outubro & 95,53 & 97,39 & 99,57 & 99,23 & 76,26 & 74,95 & 75,17 \\
\hline
\end{tabular}

Fonte: International Coffe Organization (2010). *US cents per Ib. 
Quanto ao preço do café robusta, apesar das oscilações em alguns meses, nota-se uma tendência ascendente ao longo do ano. De fato, o preço do café robusta no mercado de Nova lorque declina de 41,32 cents/lb, em janeiro de 2004, para 38,04 cents/lb, em maio de 2004. Em seguida, o preço do robusta tem uma alta, em junho de 2004, porém daí em diante apresenta uma tendência de queda de preço do café robusta com pequenas oscilações entre os meses de julho a dezembro.

Por conta da recessão econômica em alguns países, a continuar os preços baixos do café, as rendas reduzidas dos produtores, e a ausência de incentivos financeiros para produzir café de boa qualidade, torna-se difícil para muitos produtores de café investir em inovações tecnológicas de melhoramento da produção e colheita, o que invariavelmente acaba afetando a qualidade do café que pode ser produzido.

Para analisar o comportamento dos preços do café verde no mercado internacional, serão utilizados dados da
International Coffe Organization sobre as cotações de preços do café nos mercados dos EUA (Nova lorque) e, também, o quantitativo mensal dos preços dos cafés brasileiro no mercado spot de Nova lorque (New York of Trade - de janeiro de 1994 a outubro de 2006, expresso em centavos de US\$ por libra-peso (cents/b), onde cada libra-peso têm 453,6 gramas).

Sobre o desenvolvimento do modelo de análise espectral, Magnago et al. (2010, p. 01) lembra que "no Brasil ainda são poucos os estudos que determinaram a análise espectral". Por isso, houve um cuidado especial ao selecionar o período supracitado, inclusive para demonstrar a importância do processo de recuperação do mercado de café pós década de noventa, o que justifica a escolha deste período para análise e avaliação.

A existência de tendência linear da série temporal é confirmada pelo comportamento dos preços do café plotados no diagrama de dispersão, como pode ser visto na Figura 1. 
Figura 1. Evolução dos preços do café no mercado internacional: 05/01/2004-05/09/2006.

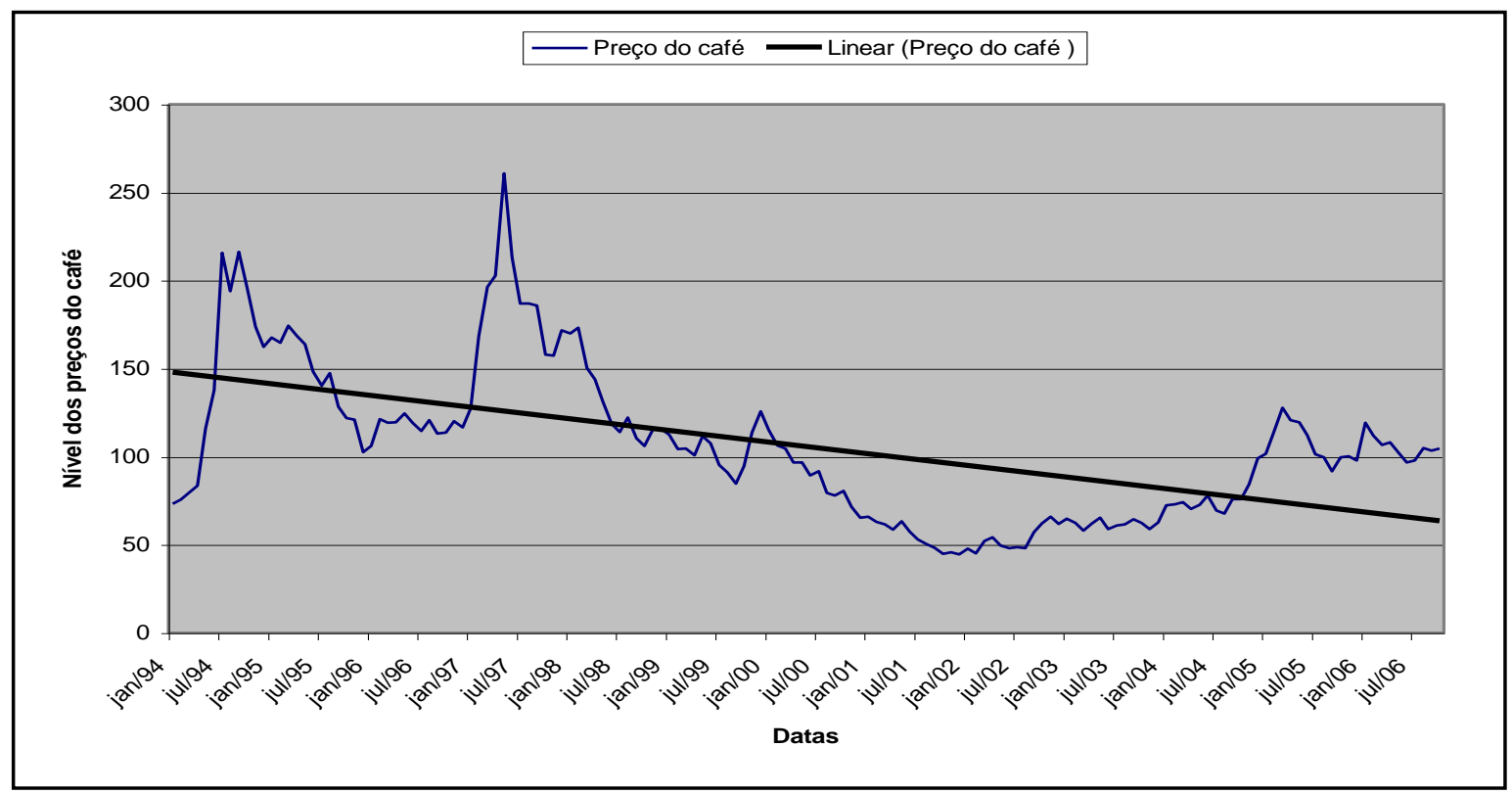

Fonte: Autor próprio.

Essa curva da cotação dos preços do café no mercado internacional de commodities agrícolas de Nova lorque apresenta um movimento sinuoso com a presença de picos e vales ao longo da trajetória de tendência linear descendente da curva dos preços do café do mercado internacional.

Nos anos de 1995 e 1996, os preços do café mantiveram-se abaixo da linha de tendência linear, mas logo apresentaram um movimento ascendente em 1997 para depois precipitar uma trajetória descendente, que vai até dezembro de 2004, com pequenas oscilações de curtíssimo prazo. Não obstante, de janeiro de 2005 a outubro de 2006, notou-se um novo movimento de recuperação ascendente dos preços do café no mercado internacional de Nova lorque que, apesar das pequenas oscilações, se mantém acima da linha de tendência linear.

Como consequência, a série temporal dos preços do café é considerada nãoestacionária, como pode ser confirmado pelos coeficientes da Função de Aucorrelação dos Preços do Café e pelas estatísticas Q, como consta na Tabela 3, cujos valores significativos a $1 \%$ de probabilidade confirmam a hipótese de que 
há pelo menos uma autocorrelação que fica fora do intervalo de confiança é aceita, como determinam Gujarati e Porter (2011) e Santos et al. (2013).

Tabela 3. Função de autocorrelação dos preços de café com 18 defasagens.

\begin{tabular}{|c|c|c|c|c|}
\hline $\begin{array}{l}\text { Defasagem } \\
\text { (LAG) }\end{array}$ & $\begin{array}{c}\text { Autocorrelação do Café } \\
\text { (ACAFE) }\end{array}$ & $\begin{array}{c}\text { Preço do Café } \\
\text { (PCAFE) }\end{array}$ & $\begin{array}{c}\text { Estatística Q } \\
\text { (Q-Stat) }\end{array}$ & $\begin{array}{l}\text { Valor de P } \\
\text { (P-Value) }\end{array}$ \\
\hline 1 & $0,9519 * \star \star$ & $0,9519 * \star \star *$ & 140,4554 & 0,000 \\
\hline 2 & $0,8975^{\star \star \star}$ & 0,0925 & 266,1228 & 0,000 \\
\hline 3 & $0,8292^{* * *}$ & $0,1737^{* *}$ & 373,0928 & 0,000 \\
\hline 4 & $0,7552^{\star * \star}$ & 0,0843 & 464,0928 & 0,000 \\
\hline 5 & $0,6944^{\star \star \star}$ & 0,1286 & 540,9527 & 0,000 \\
\hline 6 & $0,6573^{* * *}$ & $0,2346^{* \star *}$ & 610,1475 & 0,000 \\
\hline 7 & $0,6284^{\star \star \star}$ & 0,0073 & 673,8125 & 0,000 \\
\hline 8 & $0,5985^{\star \star \star}$ & $0,1513^{*}$ & 731,9482 & 0,000 \\
\hline 9 & $0,5736^{\star * \star}$ & 0,0057 & 785,7268 & 0,000 \\
\hline 10 & $0,5402^{\star \star \star}$ & 0,0289 & 833,7533 & 0,000 \\
\hline 11 & $0,5025^{\star \star \star}$ & 0,018 & 875,5942 & 0,000 \\
\hline 12 & $0,4614^{\star \star \star}$ & 0,0409 & 911,1080 & 0,000 \\
\hline 13 & $0,4215^{\star \star \star}$ & 0,0214 & 940,9578 & 0,000 \\
\hline 14 & $0,3837^{* * *}$ & 0,0025 & 965,8609 & 0,000 \\
\hline 15 & 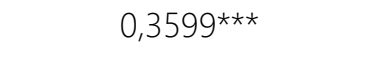 & 0,1212 & 987,9322 & 0,000 \\
\hline 16 & $0,3392^{* * *}$ & 0,0235 & 1007,678 & 0,000 \\
\hline 17 & $0,3260^{* * *}$ & 0,0048 & 1026,043 & 0,000 \\
\hline 18 & $0,3287 * \star \star *$ & $0,1201^{*}$ & 1044,854 & 0,000 \\
\hline
\end{tabular}

Fonte: Autor próprio.

Para a verificação da possibilidade da não-estacionariedade da série temporal dos preços do café, bem como para identificar se a tendência da série temporal é do tipo determinística ou estocástica, é recomendável se realizar o teste de DickeyFuller Ampliado (DFA), por ser este teste mais rigoroso e completo de forma a permitir a identificação do tipo de tendência na série temporal dos preços do café - se determinista, estocásticas ou uma combinação das duas. Nas palavras de Carvalho et al. (2007, p. 52):

Há várias maneiras de testar a presença de raiz unitária. A ênfase aqui, inicialmente, é dada ao uso da abordagem de Dickey-Fuller (DF) ou Dickey-Fuller Ampliado (DFA) como 
uma técnica para testar a hipótese nula segundo a qual, se uma série possui uma raiz unitária, então essa série é não-estacionária

O teste de DFA possui a seguinte fórmula geral:

$$
\Delta \mathrm{y}_{\mathrm{t}}=\mu+\beta \mathrm{t}+(\theta-1) \mathrm{y}_{\mathrm{t}-1}+\sum_{\mathrm{k}=1}^{\rho-1} \phi_{\mathrm{k}} \Delta \mathrm{y}_{\mathrm{t}-\mathrm{k}}+\varepsilon_{\mathrm{t}}
$$

Onde $\Delta$ é um operador de diferenças, $\mu$ e $\beta$ se referem à presença de elementos determinísticos - intercepto e tendência linear - no modelo, $\theta$ se refere à presença de raiz unitária no processo $y_{t}$ e $\rho$ é o número de lags (defasagens) incluídos na equação para incorporar a possibilidade de que $\mathrm{y}_{\mathrm{t}}$ siga a trajetória de um processo auto-regressivo de ordem superior, ressalta Greene (2017).

O teste de DFA consiste na hipótese nula conjunta $\mu=\beta=(\theta-1)=0$ para a equação (8). Se $(\theta-1)=0$, isto significa que a série possui raiz unitária, sendo considerada um processo do tipo passeio aleatório, e portanto $\mathrm{y}_{\mathrm{t}}$ apresenta somente $\mathrm{O}$ componente de tendência do tipo estocástica.
Agora, se $\mu \neq 0, \beta=0$, e $(\theta-1)=0$, então a série apresenta raiz unitária e possui o componente determinístico de intercepto, porém não possui tendência linear do tipo determinística - pois trata-se de um processo do tipo passeio aleatório com intercepto - e $y_{t}$ possui o componente de tendência do tipo estocástica.

Se $\mu \neq 0, \beta \neq 0$, e $(\theta-1)=0$, tem-se que a série apresenta raiz unitária, possui o componente determinístico de intercepto e a tendência linear determinística que definem um processo do tipo passeio aleatório com tendência linear e intercepto. Os resultados deste caso, em que $(\theta-1)=0$, indicam que a série $\mathrm{y}_{\mathrm{t}}$ deve ser considerada um processo estacionário por diferença (PED).

Para os casos em $(\theta-1) \neq 0$, em que a série temporal não possui raiz unitária e não é do tipo PED, tem-se um caso de um processo estacionário em tendência (PET), como destacado por Greene (2017). Os valores críticos dos testes feitos para a série de preços do café, visando a determinação da existência ou não-existência de raízes 
unitárias, estão sistematizados na Tabela 4

da seguinte forma:

Tabela 5. Resultados do modelo de tendência determinística dos preços do café.

\begin{tabular}{|c|c|c|c|c|}
\hline \multicolumn{5}{|c|}{ Variável Dependente: PCAFE } \\
\hline \multicolumn{5}{|c|}{ Método: Mínimos Quadrados } \\
\hline \multicolumn{5}{|c|}{ Date: 26/04/2017 Time: 06:41 } \\
\hline \multicolumn{5}{|c|}{ Amostra: 1994:01 2006:10 } \\
\hline \multicolumn{5}{|c|}{ Observações Incluídas: 154} \\
\hline Variável & Coeficiente & Erro Padrão & Estatística T & Probabilidade \\
\hline C & 177.0654 & 8.266787 & 21.41889 & 0.0000 \\
\hline $\mathrm{T}$ & -1.652776 & 0.246241 & -6.712023 & 0.0000 \\
\hline $\mathrm{T} 2$ & 0.007114 & 0.001539 & 4.623269 & 0.0000 \\
\hline$R^{2}$ & 0.403624 & \multicolumn{2}{|c|}{ Mean dependent var } & 105.7662 \\
\hline Adjusted R-squared & 0.395725 & \multicolumn{2}{|c|}{ S.D. dependent var } & 43.42039 \\
\hline S.E. of regression & 33.75290 & \multicolumn{2}{|c|}{ Akaike info criterion } & 9.895298 \\
\hline Sum squared resid & 172028.0 & \multicolumn{2}{|c|}{ Schwarz criterion } & 9.954459 \\
\hline Log likelihood & -758.9379 & \multicolumn{2}{|c|}{ F-statistic } & 51.09799 \\
\hline Durbin-Watson stat & 0.156529 & \multicolumn{2}{|c|}{ Prob(F-statistic) } & 0.000000 \\
\hline
\end{tabular}

Fonte: Autor próprio.

Pode-se observar que os valores apresentados, de maneira geral, indicam presença de raiz unitária, já que as estatísticas $\tau_{D F E}$ são maiores do que o valor crítico ao nível de significância de $1 \%$ de probabilidade, o que sugere que a série em questão é não estacionária na presença de intercepto e tendência, como se pode notar pela significância estatística dos termos $(\theta-$ 1) e $\beta$, expressa pelos valores de probabilidade.
Confirma-se, assim, que os choques climáticos sobre os preços do café poderão ter efeitos mais longos e que políticas agrícolas e macroeconômicas que afetem diretamente a cadeia produtiva do café poderão ter efeitos mais duradouros. Ademais, os resultados indicam também que a tendência dos preços do café se configura numa combinação de tendências dos tipos determinística e estocástica, o que 
sugere uma modelagem desses dois tipos de tendências presentes na série.

Em conformidade com Lamounier (2007, p. 14), "a característica essencial de uma tendência determinística se refere ao fato de que a variação no nível médio de uma dada variável se dará, de forma previsível, como uma função do tempo". Pode-se, inicialmente, estimar a parte da tendência determinística por intermédio de um modelo polinomial que, de maneira geral, pode ser expresso pela seguinte equação:

$$
\mathrm{y}_{\mathrm{t}}=\phi_{0}+\phi_{1} \mathrm{t}+\phi_{2} \mathrm{t}^{2}+\ldots \ldots \ldots+\phi_{\mathrm{k}} \mathrm{t}^{\mathrm{k}}+\varepsilon_{\mathrm{t}}
$$

Onde k é o grau do polinômio em questão; $\mathrm{y}_{\mathrm{t}}$ é uma série de tempo dos preços do café; t é a componente de tendência e $\theta$ são os coeficientes do polinômio. Na série dos preços do café, o modelo polinomial quadrático da equação (9) foi o que melhor se ajustou. A aplicação do modelo polinomial de tendência determinista quadrática é dada pela equação (9), estimado por MQO, conforme visto na Tabela 4.

$$
y_{t}=177.0654-1,652776 t+0,007114 t^{2}
$$

Valor-p $(0,000) \quad(0,000) \quad(0,000)$

$\overline{\mathrm{R}}^{2}=0,4036$

$F=51,098$

Observa-se que os coeficientes associados ao termo de tendência do tempo de grau 1 e de grau 2 são significativos a $1 \%$ de probabilidade, onde o termo de intercepto também se apresenta como significativo e, por fim, os valores estimados para os parâmetros do modelo polinomial indicam que a tendência determinística dos preços de café possui a forma de uma parábola.

Deste modo, o nível médio dos preços de café apresenta, inicialmente, um comportamento ascendente com taxas crescentes, depois o processo é revertido com um comportamento descendente com taxas decrescentes e no final da série voltase a ter um crescimento com taxas crescentes.

Figura 2. Tendência dos preços do café: residual, real e corrigida. 


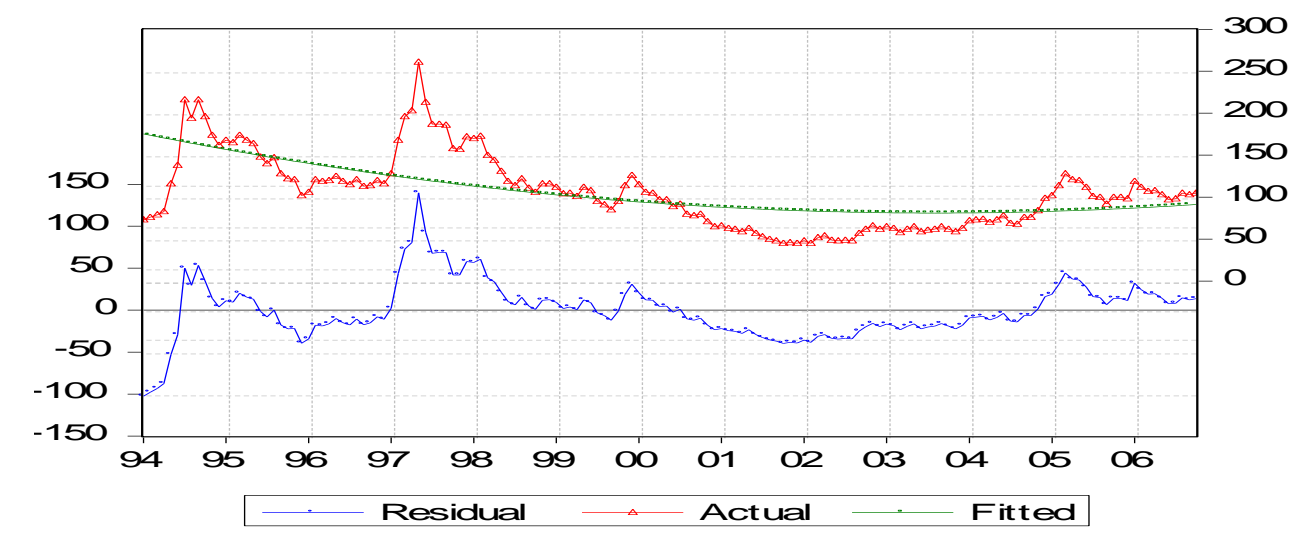

Fonte: Autor próprio.

Pode-se tomar como ponto de dos preços de café, deve-se remover referência, para prosseguir à análise de também a componente de tendência tendência, os resíduos da equação estocástica".

polinomial quadrática que melhor representou a tendência determinística da série temporal. Entretanto, na concepção de Lamounier e Leite (2007, p. 622), "para se implementar a análise espectral da série

Após o teste de raiz unitária $(A D F)$ da Figura 3, estimou-se um modelo tipo $\operatorname{ARIMA}(2,1,2)$ como o mais ajustado pelo Critério de Informação de Akaike (Akaike Info Criterion), como consta na Figura 4.

Figura 3. Teste de raiz unitária (ADF).

\begin{tabular}{|c|c|c|c|c|}
\hline ADF Test Statistic & -7.200817 & $\begin{array}{ll}1 \% & \text { Critica } \\
5 \% & \text { Critica } \\
10 \% & \text { Critica } \\
\end{array}$ & $\begin{array}{l}\text { Value* } \\
\text { Value } \\
\text { Value }\end{array}$ & $\begin{array}{l}-4.0224 \\
-3.4407 \\
-3.1446 \\
\end{array}$ \\
\hline \multicolumn{5}{|c|}{$\begin{array}{l}\text { "Mackinnon critical values for rejection of hypothesis of a unit root. } \\
\text { Augmented Dickey-Fuller Test Equation } \\
\text { Dependent Variable: D(PCAFE,2) } \\
\text { Method: Least Squares } \\
\text { Date: } 26 / 04 / 2017 \text { Time: } 07: 46 \\
\text { Sample(adjusted): 1994:07 2006:10 } \\
\text { Included observations: } 148 \text { after adjusting endpoints } \\
\end{array}$} \\
\hline Variable & Coefficient & Std Error & t-Statistic & Prob. \\
\hline $\begin{array}{l}\text { D(PCAFE (-1)) } \\
\text { D(PCAFE }(-1), 2) \\
\text { D(PCAFE }(-2), 2) \\
\text { D(PCAFE (-3),2) } \\
\text { D(PCAFE }(-4), 2) \\
\text { C }\end{array}$ & $\begin{array}{r}-1.099 \\
0.094 \\
0.271 \\
0.362 \\
0.249 \\
-0.729\end{array}$ & $\begin{array}{l}0.152639 \\
0.136377 \\
0.123977 \\
0.108160 \\
0.079187 \\
2.192685 \\
\end{array}$ & $\begin{array}{r}-7.2 \\
0.6 \\
2.1 \\
3.3 \\
3 . \\
-0.3\end{array}$ & $\begin{array}{l}000 \\
893 \\
302 \\
010 \\
020 \\
399\end{array}$ \\
\hline C & -0.729 & 2.192685 & $\frac{-0 .}{0 .}$ & $\frac{399}{824}$ \\
\hline $\begin{array}{l}\text { R-squared } \\
\text { Adjusted R-squared } \\
\text { S.E. of regression } \\
\text { Sum squared resid } \\
\text { Log likelihood } \\
\text { Durbin-Watson stat }\end{array}$ & $\begin{array}{r}0.539121 \\
0.519509 \\
12.60632 \\
22407.63 \\
-581.4788 \\
1.874194\end{array}$ & $\begin{array}{l}\text { Mean deper } \\
\text { S.D. depen } \\
\text { Akaike info } \\
\text { Schwarz cri } \\
\text { F-statistic } \\
\text { Prob(F-stati }\end{array}$ & $\begin{array}{l}\text { lent var } \\
\text { nt var } \\
\text { iterion }\end{array}$ & $\begin{array}{r}-0.135135 \\
18.18636 \\
7.952416 \\
8.094176 \\
27.48948 \\
0.000000 \\
\end{array}$ \\
\hline
\end{tabular}

Fonte: Autor próprio.

Figura 4. Resultados do modelo ARIMA $(2,1,2)$. 


\begin{tabular}{|c|c|c|c|c|}
\hline \multicolumn{5}{|c|}{$\begin{array}{l}\text { Dependent Variable: D(PCAFE) } \\
\text { Method: Least Squares } \\
\text { Date: } 26 / 04 / 2017 \text { Time: } 01: 50 \\
\text { Sample(adjusted): 1994:04 2006:10 } \\
\text { Included observations: } 151 \text { after adjusting endpoints } \\
\text { Convergence achieved after } 15 \text { iterations } \\
\text { Backcast: } 1994: 02 \text { 1994:03 }\end{array}$} \\
\hline Variable & Coefficient & Std. Error & t-Statistic & Prob. \\
\hline $\mathrm{C}$ & 0.226563 & 1.302703 & 0.173917 & 0.8622 \\
\hline $\mathrm{AR}(1)$ & 0.415280 & 0.124659 & 3.331324 & 0.0011 \\
\hline $\operatorname{AR}(2)$ & -0.513510 & o.121594 & -4.223151 & 0.0000 \\
\hline MA(1) & -0.401680 & 0.085698 & -4.687155 & 0.0000 \\
\hline MA(2) & 0.792199 & 0.087095 & 9.095758 & 0.0000 \\
\hline $\begin{array}{l}\text { R-squared } \\
\text { Adjusted R-squared }\end{array}$ & $\begin{array}{l}0.117467 \\
0.093288\end{array}$ & \multicolumn{2}{|c|}{$\begin{array}{l}\text { Mean dependent var } \\
\text { S.D. dependent var }\end{array}$} & $\begin{array}{l}0.172185 \\
13.33905\end{array}$ \\
\hline S.E. of regression & 12.70163 & \multirow{2}{*}{\multicolumn{2}{|c|}{$\begin{array}{l}\text { Akaike info criterion } \\
\text { Schwarz criterion }\end{array}$}} & 7.953890 \\
\hline Sum squared resid & 23554.39 & & & 8.053800 \\
\hline Log likelihood & -595.5187 & \multicolumn{2}{|l|}{ F-statistic } & 4.858211 \\
\hline Durbin-Watson stat & 1.959308 & \multicolumn{2}{|c|}{ Prob(F-statistic) } & 0.001046 \\
\hline $\begin{array}{l}\text { Inverted AR Roots } \\
\text { Inverted MA Roots }\end{array}$ & $\begin{array}{l}.21-.69 i \\
.20+.87 i \\
\end{array}$ & \multicolumn{2}{|l|}{$\begin{array}{l}21+.69 i \\
20-87 i\end{array}$} & \\
\hline
\end{tabular}

Fonte: Autor próprio.

Uma vantagem da abordagem da análise espectral é que este modelo possibilita ao pesquisador a busca de explicações teóricas para o comportamento observado da série analisada. Adiantou-se, no início deste artigo, que as flutuações cíclicas dos preços do café são, em geral, devidas as condições de natureza climáticas (secas e geadas) e a política do comércio exterior.

A partir daqui, busca-se resgatar, de forma objetiva, a teoria das "crises comerciais" de Jevons (1878) como uma explicação plausível para a dinâmica cíclica dos preços de café no mercado internacional. Posteriormente, complementa-se a análise com incorporação de outros ingredientes ligados a política do comercio exterior do café.
Deve-se a Jevons (1878) uma das primeiras tentativas de explicação das flutuações dos preços agrícolas devido as estações climáticas, mais do que nos fenômenos industriais. A teoria de Jevons é bastante razoável para explicar o ciclo agrícola, mas não o suficiente para explicar o ciclo econômico em geral. De fato, as mudanças climáticas que afetam as colheitas agrícolas têm claros reflexos, mesmo nas condições atuais, sobre as flutuações dos estoques das commodities agrícolas, constituindo-se assim uma das principais causas das variações dos investimentos na agricultura.

Quando a colheita é excepcionalmente abundante, em geral, há uma adição relevante ao estoque regulador dos preços para os anos futuros. Os resultados dessa 
adição acrescentam-se à renda corrente dos agricultores e são considerados, por parte dos próprios agricultores, como renda desde que esse incremento não implique em vazamento na forma de gastos para os outros setores da economia, senão quando financiados pela poupança.

De modo semelhante, quando a colheita é bem escassa, recorre-se aos estoques armazenados para atender ao consumo corrente, de tal maneira que uma parcela correspondente dos gastos que os consumidores fazem de suas rendas não cria nenhuma renda corrente para os agricultores. Isto quer dizer que a parte que é retirada dos estoques implica numa redução correspondente do investimento no setor agrícola.

Nestas condições, na hipótese de que o investimento nos demais setores da economia for constante, pode ser grande a diferença no investimento agregado num período em que haja uma adição substancial aos estoques conservados; e num outro em que tenha havido uma redução substancial dos estoques. Assim, numa economia de mercado onde a agricultura tenha um peso bastante relevante, é razoável supor que as flutuações dos estoques sejam uma das causas das variações dos investimentos e dos preços na agricultura com reflexos na economia como um todo.

Logo, é plausível que a reversão das fases ascendentes da série analisada seja indicada por boas colheitas resultantes da expansão da produção, inclusive com a inserção de novos concorrentes como no caso do Vietnã, que elevaram o nível dos estoques e precipitaram uma queda dos preços correntes com reflexos nos preços futuros. Há quem diga que seriam as más colheitas - e não as boas - que incitam mais o comércio agrícola porque motivam a mão-de-obra agrícola a aceitar um salário real menor, ou porque se considere que uma mudança na distribuição de renda seja favorável ao consumo de bens agrícolas.

Não obstante esses argumentos, há de convir que as causas das flutuações agrícolas dos preços têm na economia contemporânea uma importância menor da que teve na época de Jevons, por pelo menos duas razões: primeiro, porque a produção agrícola nos dias atuais representa uma parcela 
pequena da produção da economia global; segundo, porque, com a ampliação do mercado mundial para a maioria dos produtos agrícolas, mercado este que se abastece de commodities agrícolas de todos os continentes, é provável que, em média, os efeitos das más e boas colheitas sejam neutralizados numa proporção menor do que nos tempos de Jevons.

Outro fator que pode ter contribuído para o comportamento cíclico dos preços do café brasileiro, entre os anos de 1994 a 2006, foi a política de comércio exterior. Além do "custo Brasil", a política cambial e a política de cotas, estabelecida nos Acordos Internacionais do Café (AIC), comprometeram a participação do Brasil internacionalmente.

No início, a política do sistema de cotas tinha como objetivo sustentar os preços do café mediante o controle da oferta. Todavia há uma concordância, entre os especialistas do mercado internacional do café, de que essa política de cotas vinha reduzindo a participação relativa das exportações brasileiras no total das exportações mundiais de café. Isto ocorreu porque a sustentação dos preços internacionais estimulou o crescimento da oferta de café de alguns países, sobretudo na Colômbia e no Vietnã.

Após a suspensão das cláusulas do AIC administrada pela Organização Internacional do Café (OIC), a economia do café entrou em crise provocando uma grande perda de renda, estimada em US\$10 bilhões, para os países produtores de café entre 1989/92. Foi preciso uma nova rodada de negociação, entre as partes interessadas, para que o tema das cotas voltasse a fazer parte da agenda dos países produtores de café, liderados pelo Brasil, que queriam ampliar suas cotas de exportações via acordos e não mais através da OIC.

\section{CONCLUSÃO}

A análise de séries temporais, por meio da decomposição dos seus componentes de tendência, ciclos, sazonalidade e volatilidade, apresenta uma série de vantagens em relação a outras metodologias, pois permite a interpretação individual dos seus componentes. De fato, os modelos estruturais de séries temporais, que consideram de forma explícita as componentes não-observáveis das séries, 
têm o mérito de forçar o pesquisador a procurar explicações teóricas para o comportamento observado de alguns componentes específicos das séries analisadas, como o caso que foi analisado do café.

Isto ocorre porque a incidência e a magnitude das componentes mencionadas, em geral, possuem explicação empírica em função das singularidades dos mercados, dos fatos econômicos e das próprias variáveis que estão sendo analisadas. Essa abordagem alternativa reduz, em parte, as críticas que os modelos das séries temporais sofrem por estarem, de certa maneira, dissociados da teoria econômica uma vez que são modelos extrapolativos e para o caso dos modelos univariados por não estabelecerem as relações de causa e efeito como geralmente ocorrem nos modelos econométricos de regressão múltipla.

Neste campo das análises estruturais de séries, as análises espectrais (domínios de frequências) dos ciclos de séries temporais constituem uma nova abordagem de análises de séries que fornece outras informações complementares quando se trata de análises de ocorrência de ciclos de séries temporais com uma razoável temporalidade. Este foi o caso da aplicação da análise espectral no comportamento dos preços do café no mercado internacional, cujos resultados obtidos foram devidamente analisados.

\section{REFERÊNCIAS}

BRUNATO, P. B.; HENNING, E.; WALTER, O. M. F. C.; TORTORELLA, G. L. Aplicação de métodos de previsão de séries temporais em uma indústria têxtil. Revista Espacios, V. 37, N. 37, 2016. Disponível em: http://www.revistaespacios.com/a16v37n37 /16373720.html. Acesso em: 19/10/2017.

CARVALHO, D. F.; RIBEIRO, M. R.; SANTANA, A. C.; CUTRIM CARVALHO, A. Análises dos testes de cointegração e de correção de erro dos preços do café e do cacau no mercado internacional de futuros e opções. Novos Cadernos NAEA, [S.I.], v. 10, n. 1, dez. 2008. ISSN 2179-7536. Disponível em: $<$ http://www.periodicos.ufpa.br/index.php/ ncn/article/view/71>. Acesso em: 19/10/2017.

CHIESA, D. D.; MIGUEL, L. F. F. Geração de uma excitação sísmica através do espectro de Kanai-Tajimi. Revista Brasileira de Computação Aplicada, [S.I.], v. 9, n. 1, p. 3141, maio 2017. ISSN 2176-6649. Disponível em: 
<http://seer.upf.br/index.php/rbca/article/v iew/6660 > . Acesso em: 19/10/2017.

CRÁMER, H; LEADBETTER, M.R. Stacionary and related stochastic processes. New York, 1967.

CUNHA, D. A.; do VALE, S. M. L. R; BRAGA, M. J. B; CAMPOS, A. C. Integração e transmissão de preços no mercado internacional de café arábica. Revista de Econonomia e Sociolologia Rural, Brasília , v. 48, n. 4, p. 515542, Dec. 2010. Disponível em: <http://www.scielo.br/scielo.php?script=sci _arttext\&pid $=$ S0103 20032010000400002\&lng =en \&nrm=iso > . Acesso em: 19/10/2017.

DIAS, L. O.; SILVA, M. S. Determinantes da demanda internacional por café brasileiro. Revista de Política Agrícola, Ano XXIV, N. 01, jan./fev./mar, 2015. Disponível em: https://ainfo.cnptia.embrapa.br/digital/bitst ream/item/126824/1/Determinantes-dademanda-internacional.pdf . Acesso em: 19/10/2017.

ESQUIVEL, R. M.; SENNA, V.; GOMES, G. S. $S$. Análise Espectral Singular: Comparação de previsões em séries temporais. Revista ADM. MADE , v. 16, p. 87-101, 2012.

GREENE, W. H. Econometric analysis. NJ: Pearson Prentice Hall, ed. 8, 2017.

GUJARATI, D. N.; PORTER, D. C. Econometria básica. Porto Alegre: Mcgraw Hill Bookman, 924p, 2011.
International Coffee Organization ICO. Historical Data. Disponível em: <http://www.ico.org/asp/select7.asp>. Acesso em: 19/10/2017.

JEVONS, W. S. Commercial Crises and SunSpots. Nature, XIX, nov 1878.

LAMOUNIER, W.M., LEITE, C. A. M. Análise estrutural de séries temporais. In: Métodos quantitativos em economia. Maurinho $L$. dos Santos e Wilson da C.Vieira. (Eds.), Viçosa, UFV, 2004.

LAMOUNIER, W. M. Tendência, ciclos e sazonalidade nos preços spot do café brasileiro na NYBOT. Revista Gestão e Produção, São Carlos, v. 14, n. 1, p. 1323, Apr. 2007 . Disponível em: <http://www.scielo.br/scielo.php?script=sci _arttext\&pid=S0104530X2007000100003\&lng=en \&nrm=iso > . Acesso em: 19/10/2017.

MAGNAGO, R; FISCH, G; MORAES, O. Análise espectral do vento no Centro de Lançamento de Alcântara (CLA). Revista Brasileira de meteorologia, São Paulo, v. 25, n. 2, p. 260-269, June 2010. Disponível em:

<http://www.scielo.br/scielo.php?script=sci _arttext\&pid=S0102-

77862010000200010\&lng =en\&nrm=iso $>$. Acesso em: 19/10/2017.

MATTOS, D. M. ; FERREIRA, P. G. C.; Usando o R para ensinar Ajuste Sazonal. Texto para Discussão n 89, Portal IBRE (IBRE|FGV), $2016 . \quad$ Disponível em: http://portalibre.fgv.br/main.jsp?lumPageld $=4028818$ B43102A940143103563A00D88\& 
contentld =8A7C82C5519A547801533DF9B

FFE3EEE. Acesso em: 19/10/2017.

MORETTIN, P. A.; TOLOI, C. M. C. Análise de séries temporais. São Paulo, Edgar Blücher, 2004.

NISHIJIMA, M.; SAES, M. S. M; POSTALI, F. A. S. Análise de concorrência no mercado mundial de café verde. Revista de Econonomia e Sociologia Rural, Brasília, v. 50, n. 1, p. 69-82, Mar. 2012 . Disponível em:

$<$ http://www.scielo.br/scielo.php?script=sci _arttext\&pid=S0103$20032012000100004 \& \operatorname{lng}=e n \& n r m=i s o>$. Acesso em: 19/10/2017.

REGO, B. R.; DE PAULA, F. O. O Mercado Futuro e a Comercialização de Café: Influências, Riscos e Estratégias com uso de Hedge. Revista Gestão \& Conhecimento. V. 7, N. 1, Artigo 1, Mar./Jul., p. 01-26, 2012. Disponível em: https://www.pucpcaldas.br/graduacao/ad ministracao/revista/artigos/v7n1/v7n1a1.pdf . Acesso em: 19/10/2017.

SANTOS, H. G.; SÁ, L. A. C. M.; NERO, M. A.; PORTUGAL; J. L. Análise exploratória espacial dos preços das terras agrícolas no estado de São Paulo. Revista Brasileira de Geomática (RBGeo), v. 3, n. 1, p. 02-11, jan./jun., 2015.

SARTORIS, A. Estatística e Introdução à Econometria. São Paulo: Saraiva, 2013.

WOLFF, L.; ALMEIDA, S. G. A.; ZANINI, R. R.; MACEDO, J. H. S.; SOUZA, A. M. Análise do Índice Bovespa sob enfoque de séries temporais. Revista Global Manager. V. 11, n. 01, 2011. 